\title{
Aspects of baryon spectroscopy at ELSA
}

\author{
Hartmut Schmieden ${ }^{1, *}$ \\ for the BGO-OD Collaboration \\ ${ }^{1}$ Physikalisches Institut, Rheinische Friedrich-Wilhelms-Universität, Bonn, Germany
}

\begin{abstract}
A renaissance in hadron specroscopy in both meson and baryon sectors was triggered by the discovery of the enigmatic $X, Y, Z$ states. Meanwhile, the discussion on unconventional quark-gluon compositions to understand their internal structure expanded to baryonic states in the charm sector, i.e. the pentaquark candidates observed at LHCb. Old problems and recent findings in the strange sector may suggest similar structures to exist there as well. In this light recent results and the ongoing new BGO-OD experiment at ELSA are discussed.
\end{abstract}

\section{Introduction}

During the 1990's, high resolution meson photoproduction experiments like A2 at MAMI, CLAS at JLab, Crystal Barrel at ELSA or LEPS at SPring-8 set out to finally solve the socalled missing resonance problem of baryon spectroscopy. It was hoped to observe baryon resonance states previously unseen in experiments using pion and kaon beams. Of particular interest were photoproduction channels with mesons other than pions, e.g. $K$ or $\omega$. To analyse the extensive high quality date sets obtained, very sophisticated PWA and isobar models were developed, e.g. BnGa [1] or MAID [2], which also were presented at this conference.

Of particular interest is the energy-mass region around $2 \mathrm{GeV}$, the second and third resoance regions, where over 30 states expected in 3-quark potential models remained unseen in experiments. Based on the comprehensive data and PWAs, new resonances could be included in the PDG tables [3] or the star-rating improved. This was the first time based on photoproduction experiments and can be viewed as a big success. On the other hand, the sheer number of new states is far below of what was to be expected after two decades of intense research. The status of nucleon states in the resonance region around $2 \mathrm{GeV}$ is summarised in Table 1.

A new chapter has opened in hadron spectroscopy since a decade ago when in the $c$ sector unexpected, narrow surplus states were discovered in the meson spectrum, $X(3872)$ the first one at Belle [4], and later in the baryon spectrum two states at LHCb [5], labelled $P_{c}^{*}(4380 / 4450)$. For both, the new mesons and baryons, the quark content must apparently exceed minimal $q \bar{q}$ and $q q q$ configurations. Interestingly, both $X(3872)$ and $P_{c}^{*}(4380 / 4450)$ are located directly at thresholds, $D^{*} D$ and $D^{*} \Lambda_{c} / \Sigma_{c}$, respectively. Since meson-baryon dynamics is expected to play an important role especially at thresholds, hadronic molecules are among the possible explanations to understand the structure of the states. Indeed, the only prediction of the $\mathrm{LHCb}$ pentaquark states was obtained in a model of vector meson - baryon interactions [6].

\footnotetext{
*e-mail: hs@physik.uni-bonn.de
} 
Table 1. Status of nucleon resonance states at around $2 \mathrm{GeV}$ mass in PDG and PWA[1].

\begin{tabular}{lclc}
\hline State & PDG 2010 & BnGa PWA & PDG 2012 \\
\hline$N(1860) 5 / 2+$ & - & $*$ & $* *$ \\
$N(1875) 3 / 2-$ & - & $* * *$ & $* * *$ \\
$N(1880) 1 / 2+$ & - & $* *$ & $* *$ \\
$N(1895) 1 / 2-$ & - & $* *$ & $* *$ \\
$N(1900) 3 / 2+$ & $* *$ & $* * *$ & $* * *$ \\
$N(2060) 5 / 2-$ & - & $* * *$ & $* *$ \\
$N(2150) 3 / 2-$ & - & $* *$ & $* *$ \\
\hline
\end{tabular}

It is an open question whether states similar to the (hidden) $c$-sector could manifest also in the hidden or open $s$-quark sector. Obviously, rich threshold dynamics are in place there as well. This is illustrated in Fig. 1. The idea of possible interactions of the light mesons

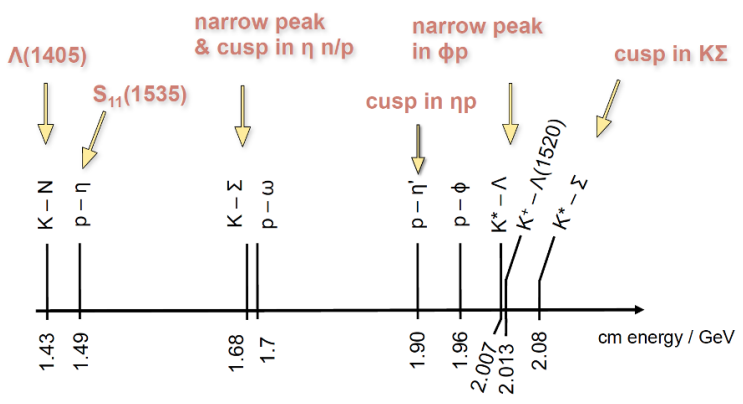

Figure 1. Overview of thresholds and associated pecularities in meson photoproduction.

directly with quarks, and the formation of meson-meson or meson-baryon type multi-quark objects dates back quite some time already. It provided means to explain issues of the baryon spectrum unresolved in genuine 3-quark models, e.g. the parity ordering of the lowest nucleon excitations [7]. Of special interest in this respect is the cusp in $K_{s}^{0} \Sigma_{\text {g.s. }}^{+}$photoproduction directly at the $K^{*}$ threshold, labelled in Fig. 1, and first observed at ELSA in two independent experiments, c.f. [8] and references therein. It can be explained through a pair of states $N^{*}(2030 / 2080)$ by the same vector meson - baryon model predicting the LHCb pentaquarks, if $c \bar{c}$ is exchanged through $s \bar{s}$ [9]. In this Reference, E. Oset and A. Ramos propose a smoking gun experiment to investigate this issue: $\gamma n \rightarrow K_{s}^{0} \Sigma_{\text {g.s. }}^{0}$ on the neutron, where the constructive interference of the two involved states is predicted to produce a peak structure as opposed to the cusp through destructive interference on the proton.

Another enigmatic state of the light baryon sector ist the $\Lambda(1405)$. It is located directly at the $\bar{K} N$ threshold, and meson-baryon dynamics apparently play a crucial role for it, see e.g. $[11,12]$. Recently, also lattice QCD results confirmed a predominantly $\bar{K} N$ molecular structure of the $\Lambda(1405)$ [13]. In either of the discussed cases, forward kaon production will play a crucial role in the photoproduction of the molecular-type states, because the momentum transfer to the loosely bound system is minimised. An experiment should therefore possess good acceptance, resolution and particle id for very forward going kaons, complemented with close to $4 \pi$ acceptance for neutral and charged products of the almost at rest resonance decays. The BGO-OD experiment at ELSA matches these requirements in an almost ideal manner. 


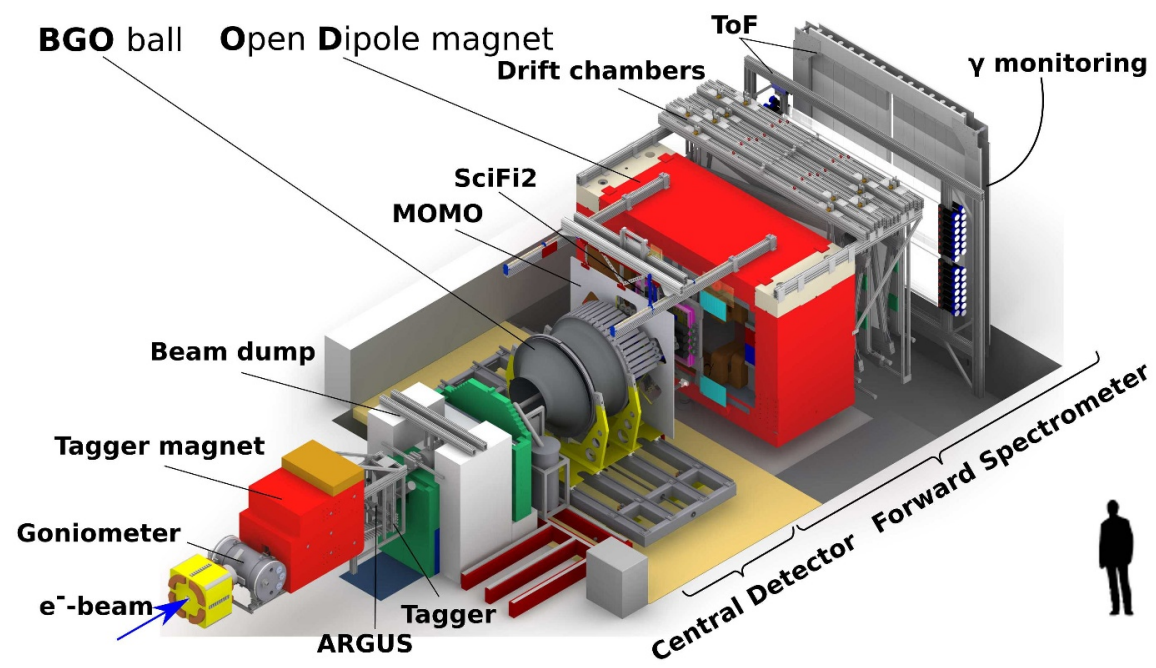

Figure 2. Overview of BGO-OD setup.

\section{BGO-OD experiment at ELSA}

The experiment is located at the electron stretcher accelerator ELSA [14] of the University of Bonn. The overall layout is sketched in Fig. 2. Bremsstrahlung is used to produce photon beams from the electron beam extracted from the stretcher ring. The photon energy is eventwise tagged by a post bremsstrahlung electron spectrometer.

The BGO-OD detector consists of two main parts, the forward magnetic spectrometer with the large aperture Open Dipole (OD) magnet ${ }^{1}$ as the main component, and the central detector based on the BGO crystal calorimeter ${ }^{2}$.

The forward magnet is sandwiched between tracking detectors and complemented with a time-of-flight (ToF) spectrometer. Front tracking is provided by two layers of scintillating fibre detectors, MOMO and SciFi2. Eight double layers of drift chambers enable tracking behind the magnet. The spectrometer achieves momentum resolutions of $\simeq 3 \%$ at $1 \mathrm{GeV} / \mathrm{c}$. Particle identification is provided by combination of momentum and time of flight measurements. A lab angular range up to $\simeq 12$ degree is covered.

Main part of the central detector is the BGO "rugby ball". It consists of 480 BGO crystals of 21 radiation lengths with individual photomultiplier readout $[17,18]$. The calorimeter is supplemented by a double layer cylindric MWPC for charged particle tracking, and a 32 segment scintillator barrel for particle id based on specific energy loss. The central detector covers the angular range $25-155$ degree. The acceptance gap between the forward hole of BGO and the magnet spectrometer is closed by SciRi, a 96 channel annular scintillator detector. For the experiments described here a cryogenic liquid hydrogen or deuterium target was operated at the centre of the BGO ball. A more detailed description of the setup and its performance can be found in [15] and [16].

\footnotetext{
${ }^{1}$ Magnet and power supply are on permanent loan from DESY, Hamburg.

${ }^{2}$ supplied by INFN Frascati and run by the Italian groups of the collaboration
} 

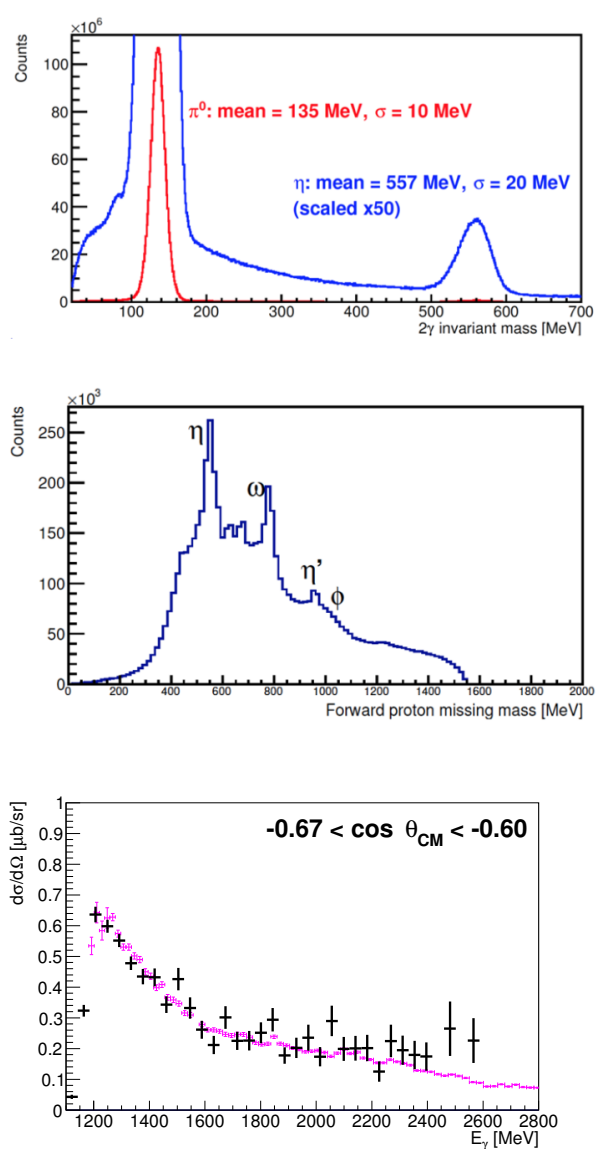

Figure 3. Two-photon invariant mass spectrum taken with the central BGO calorimeter.

Figure 4. Missing mass spectrum optained from tagged beam photon and forward proton measured in the OD spectrometer.

Figure 5. Preliminary differential cross section of the reaction $\gamma p \rightarrow \omega p$ for the $\omega \mathrm{cm}$ angular bin inset. Black data is BGO-OD, in comparison to CLAS [19] (cyan).

\section{Preliminary results with the BGO-OD experiment}

After commissioning, the BGO-OD detector is used for routine data taking since 2016. In the following first preliminary results are presented.

Fig. 3 shows the two photon invariant mass distribution reconstructed with the central calorimeter. Peaks of $\pi^{0}$ and $\eta$ mesons are clearly visible with achieved resolutions inset. Fig. 4 presents the missing mass distribution calculated from initial beam photon and final state proton identified in the forward spectrometer. Clearly visible are the recoiling narrow mesons, $\eta$ and $\omega$, and also $\eta^{\prime}$ and $\phi$ can be identified.

From the identified non-strange channels, bench-mark differential cross section have been determined. As an example, one bin of $\omega$ photoproduction is shown in Fig. 5. Reasonable agreement is obtained with previous ultra-high statistics data from CLAS [19].

In analogy to the proton in Fig. 4, the missing mass obtained from $K^{+}$identified in the forward spectrometer is shown in Fig. 6. Ground and excited state hyperons clearly pop up immediately, without further analysis. Based on this it is possible to extract $K^{+}$photoproduction cross sections in forward angular region previously unaccessible by most experiments. Examples are shown in Fig. 7 for associated $\Sigma^{0}$ (top) and $\Lambda$ (bottom) production. Especially in the $K^{+} \Lambda$ channel, our new data will resolve the discrepancy between existing data sets and constrain PWAs and isobar models.

In Fig. 6 the $\Lambda(1405)$ shows up, superimposed by the $\Sigma^{0}(1385)$. In principle, both can be separated through the pure isospin $I=0$ decay channel $\pi^{0} \Sigma_{\text {g.s. }}^{0}$ which is allowed for the 

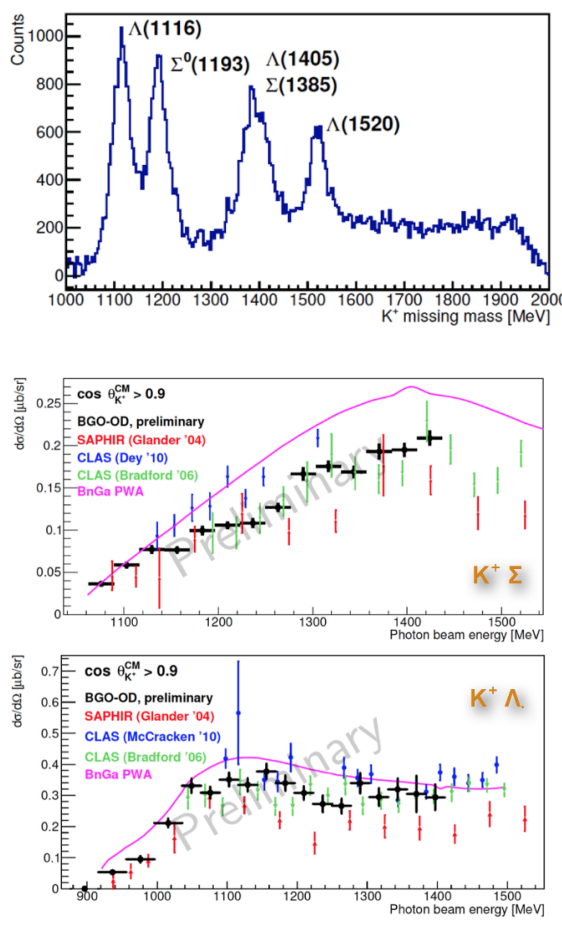

Figure 6. Missing mass to the $K^{+}$identified in the forward spectrometer and the tagged photon.

Figure 7. Preliminary differential cross sections for the reactions $\gamma p \rightarrow K^{+} \Sigma_{\text {g.s. }}^{0}$ (top) and $\gamma p \rightarrow K^{+} \Lambda_{\text {g.s. }}$. (bottom). Forward going kaons are detected in the OD spectrometer. Black points represent the preliminary new BGO-OD data. For comparison older CLAS data is plotted in green [20] and blue [21], and SAPHIR in red [22] . The magenta line shows the present BnGa partial wave fit, essentially to the newer CLAS data. Lacking most forward acceptance, CLAS data is in the $\cos \theta_{K^{+}}^{c m}$ 0.85 to 0.95 interval.

$\Lambda(1405)$ but forbidden for the $\Sigma^{0}(1385)$. The $\Sigma_{\text {g.s. }}^{0}$ has a $100 \%$ decay into $\Lambda_{\text {g.s. }} \gamma$ and, hence, the $\Lambda(1405)$ is expected to show up in the $\pi^{0} \Lambda \gamma$ invariant mass distribution, sometimes called the "line shape". Fig. 8 shows corresponding distributions, separated into two angular regions of the associated $K^{+}$. In addition to existing comprehensive data sets from CLAS [23], it is possible to access $\Lambda(1405)$ production at lowest possible momentum transfer, i.e. very forward going $K^{+}$.
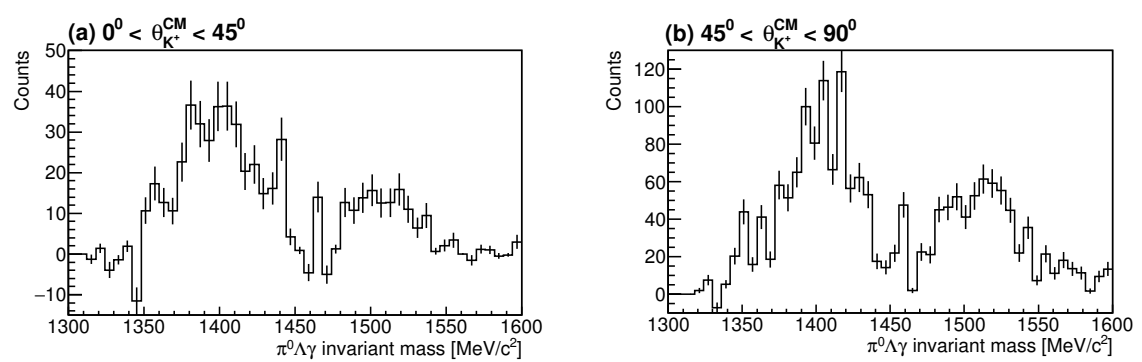

Figure 8. Very preliminary invariant mass distributions in the pure isospin $I=0$ channel $\pi^{0} \Sigma^{0} \rightarrow \pi^{0} \Lambda \gamma$ in the mass range of the $\Lambda(1405)$, split into two angular regions of forward going $K^{+}$in the reaction $\gamma p \rightarrow K^{+} X$.

As motivated in the introduction, a very interesting channel to measure is $\gamma n \rightarrow K_{s}^{0} \Sigma_{\text {g.s. }}^{0}$. due to its predicted sensitivity to vector meson-baryon dynamics in analogy to the $c$-quark sector. Therefore, two days test data was taken using a liquid deuterium target. After cuts to ensure the event topology of the neutral reaction, the $K^{0}$ shows up in the $\pi^{0} \pi^{0}$ invariant mass distribution, c.f. Fig. 9. The same analysis on a liquid hydrogen target produced no 
visible $K^{0}$ signal. A detailed analysis will be performed on an extensive data set taken after the conference.

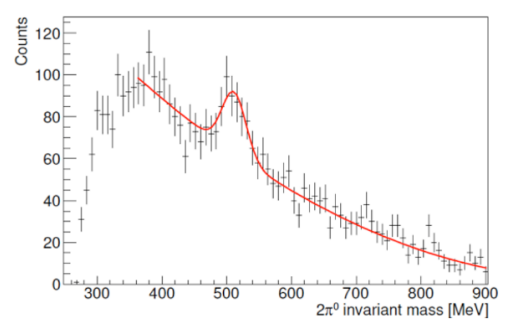

Figure 9. $\pi^{0} \pi^{0}$ invariant mass distribution obtained from a deuteron target with cuts on the topology of the reaction $\gamma n \rightarrow K_{s}^{0} \Sigma^{0}$. The $K_{s}^{0}$ exhibits itself as a clear enhancement. The proton of the decay $\Sigma^{0} \rightarrow p \pi^{0}$ was required in the forward spectrometer. With the cuts applied no $K^{0}$ signal is visible with proton target.

In summary, BGO-OD is a unique experiment for meson photoproduction, especially suited for forward kinematics and low momentum transfer processes. This is particularly interesting for associated kaon-hyperon production, and first preliminary results have been presented.

We thank DESY for providing magnet and power supply of the OD forward spectrometer, and IPN Orsay for scintillator detectors of the time-of-flight spectrometer. This work is supported by DFG under project PN 50165297.

\section{References}

[1] A. V. Anisovich et al., Eur. Phys. J. A 50, 129 (2014)

[2] see L. Tiator, Few-Body Syst. 59, 21 (2018), and references therein

[3] C.Patrignani et al., Chin Phys. C 40, 100001 (2016)

[4] S.-K. Choi et al. (Belle Collaboration), Phys. Rev. Lett. 114, 262001 (2003)

[5] R. Aaij et al. (LHCb Collaboration), Phys. Rev. Lett. 110, 222001 (2013)

[6] J.-J. Wu, R. Molina, E. Oset, and B. S.Zou, Phys. Rev. Lett. 105, 232001 (2010)

[7] L.Ya. Glozman and D.O. Riska, Physics Reports 268, 263 (1996)

[8] R. Ewald et al., Phys. Lett. B 713, 180 (2012)

[9] A. Ramos and E. Oset, Phys. Lett. B 727, 287 (2013)

[10] J.M.M. Hall et al., Phys. Rev. Lett. 114, 132002 (2015)

[11] D. Jido, J.A. Oller, E. Oset, A. Ramos, U.-G. Meissner, Nucl. Phys. A 725, 181 (2003),

[12] M. Mai, U.-G. Meissner, Nucl. Phys. A 900, 51 (2013),

[13] J.M.M. Hall et al., Phys. Rev. Lett. 114, 132002 (2015)

[14] W. Hillert, Eur. Phys. J. A 28, s01, 139 (2006)

[15] H. Schmieden et al. (BGO-OD Collaboration), Few-Body Syst. 59, 135 (2018)

[16] T. C. Jude et al. (BGO-OD Collaboration), arXiv:1808.10622 [nucl-ex], submitted proceedings for The 13th International Conference on Hypernuclear and Strange Particle Physics (2018)

[17] F. Ghio et al., Nucl. Instr. Meth. A 404, 71 (1998)

[18] P. Levi Sandri et al., Nucl. Instr. Meth. A 370, 396 (1996)

[19] M. Williams et al., Phys. Rev. C 80, 065208 (2009)

[20] R. Bradford et al., Phys. Rev. C 73, 035202 (2006)

[21] M. E. McCracken et al., Phys. Rev. C 81, 025201 (2010)

[22] K. H. Glander et al., Eur. Phys. J. A 19, 251 (2004)

[23] K. Moriya et al., Phys. Rev. C 87, 035206 (2013) 